\title{
A.R.S. - Art Resistance Shoah: la memoria attraverso l'arte
}

L'articolo presenta il nuovo progetto di Istoreco sulla memoria storica vista dalle arti visive che commemorano Resistenza e Shoah.

The paper deals with the new project of Istoreco about memory seen from the visual art commemorating Resistance and Shoah.

Fondato da chi scrive e da Margherita Fontanesi nel 2012, con il patrocinio culturale e l'incoraggiamento intellettuale dell'Istoreco di Reggio Emilia, A.R.S. - Art Resistance Shoah non ricerca significati astratti nell'arte, ma promuove ogni produzione artistica che si sia impegnata o s'impegni sui temi di commemorazione della Resistenza e della Shoah. Dallo scorso marzo A.R.S. è entrato a far parte ufficialmente dei progetti di Istoreco, con l'ingresso della storica dell'arte Elisabetta Del Monte, avviandosi alla realizzazione di una grande mostra collettiva in memoria della Resistenza per il $70^{\circ}$ della Liberazione, che avrà come partner anche l'Anpi di Reggio Emilia.

Dato pregnante di questo progetto è la foce della memoria, quali esiti e sviluppi possano esserci nella divulgazione e per la società, partendo da un postulato: i testimoni oculari, spesso, per raggiunti limiti di età, non riescono più a raccontare. Ora tocca ai testimoni dei testimoni.

L'empatia è la dote connaturata all'individuo, giunge - se trasfusa nell'ispirazione d'artista - ad apici di arricchimento, d'analisi e dunque d'estrema necessità, utili a farsi carico della memoria, in tempi molto difficili e culturalmente in pericolo. Sondare la storia dell'arte significa anche, da sempre, studiare e prendere atto 
degli avvenimenti, accorgendosi anche quanto l'arte stessa, oltre che per diletto, abbia preso parte in modo totalizzante alle scelte politiche nella storia. Per il nazifascismo fu programma di persuasione e motivo cardine. Carpire certi nessi è più facile attraverso l'arte, ma stupisce sempre anche noi, il suo non essere stato un processo elettivo intuitivo, a quanto pare. Con risultati d'eccezione quando sia illuminante maturare con l'arte che affronti percorsi visivi di ammonimento, narrazione e commemorazione. Da questo punto di vista, A.R.S. era l'elemento mancante nel panorama di studi e analisi intorno ai temi del nazifascismo europeo e della commemorazione, attestandosi come primo progetto nazionale che prenda in seria considerazione a livello programmatico la produzione d'arte per la memoria storica.

\section{Oblio e delirio}

La nascita di un simile progetto, richiede però alcuni elementi propedeutici di narrazione, specie per capire la mole di riferimento quanto grande possa essere. Tirare un sospiro di sollievo rispetto alla posizione delle arti visive in ambito politico e al rischio di annichilimento precorso con il nazionalsocialismo in Germania e il fascismo, in Italia pare doveroso. La mostra allestita a Correggio dal 27 gennaio al 10 febbraio 2014, organizzata proprio da A.R.S. con il patrocinio dell'Istoreco

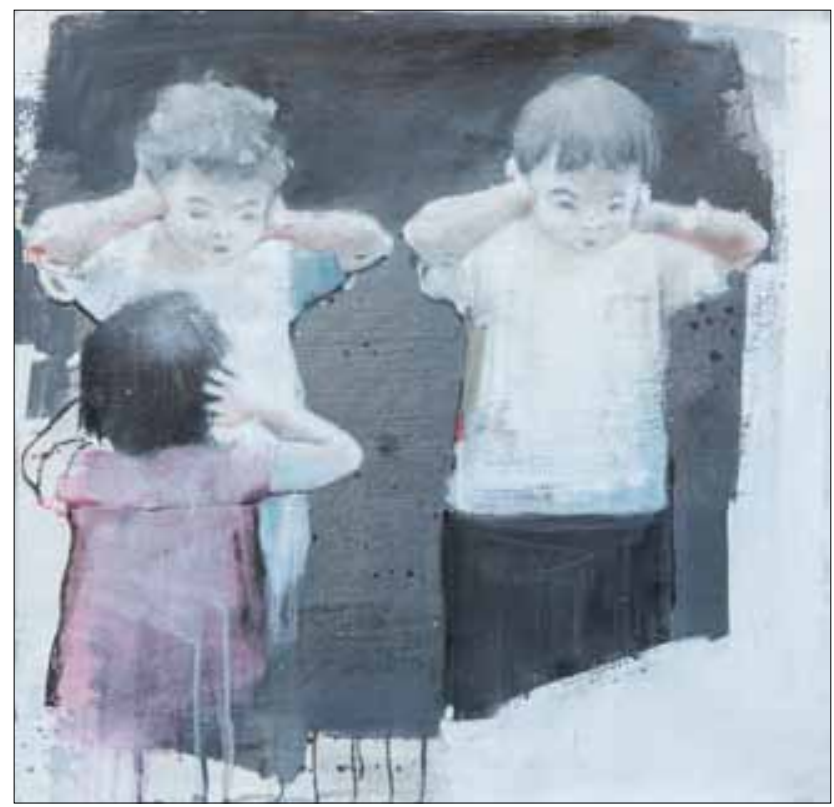

Dalla mostra In Absentia: Massimo Lagrotteria, Senza titolo (2013) e del Comune di Correggio, ha avuto questa funzione e per questo aveva il titolo evocativo In Absentia.

E dopo la mostra quel che appunto occorre ricordare è la riflessione su quel che ci manca, su quel buco umano e intellettuale voluto dal nazifascismo. Un buco terribile che produce sgomento ogniqualvolta si riflette non solo sui milioni di morti, ma su quanti di essi avrebbero dato all'Europa il bene 
di un segno nelle arti, cultura, medicina, legge, etc.

Un'orrenda punizione per tutto il nostro continente. Come poter parlare attraverso l'arte "in assenza" (!) dei testimoni integrali, perché periti nelle camere a gas subito al loro arrivo nei centri di messa a morte dell'Europa dell'Est? Tradizione culturale e empatia contengono a gradi differenti nessi e risposte. Ci viene incontro un dato intellettuale, importante e ineludibile, che il fascismo europeo è figlio legittimo e primo ereditario della storia del "vecchio continente". È cresciuto e si è formato dentro società parecchio avanzate, che non hanno fatto per nulla a meno di una tradizione culturale ben radicata.

L'arte tradizionale è stata costretta dai nazisti e fascisti a andare con i soldati all'attacco, a farsi portavoce d'ideali razzisti e esclusivisti in nome di un'impalcatura che tenesse serrati i ranghi dell'orrore e del terrore. Il fascismo è frutto del positivismo sfrenato ed è sfociato per questo motivo molto velocemente in recrudescenze di violenza pianificata. Distruzioni, persecuzioni, schiavismo, genocidi, occultamento delle prove, se non guerre, sono fenomeni che di epoca in epoca, lungi dall'essersi estinti, con il fascismo hanno raggiunto apici culmine.

E se non si può sostenere di aver conquistato la cima di un monte, senza averlo scalato partendo dal suo punto più a valle, è altrettanto innegabile che la scomparsa di tracce tangibili - a parte le ceneri dei cadaveri - e il segreto assoluto intorno ai campi di sterminio, pesano non solo come un violento atto criminale, ma anche come un perfido progetto in continuità storica europea per cancellare ogni segno culturale ebraico. Un odio verso l'ebreo, connaturato, che ha trovato in Hitler e Mussolini i più raffinati esponenti storici. A una più precisa analisi sulle scelte politiche nazifasciste, infatti, rispetto alla questione razziale, si evince un polo comprimario del medesimo quadro: la codifica di nuovi linguaggi espressivi "ariani" sia in Germania sia in Italia, come riempitivi del vuoto in arrivo con la soluzione finale della questione ebraica, chiamato modernamente Shoah e conosciuto, col termine improprio, ma ben radicato, di Olocausto.

Un fatto saltato agli onori della cronaca lo scorso 4 novembre 2013, a Monaco di Baviera, chiarisce più di ogni altro esempio lo stato delle cose. La polizia di frontiera tedesca ha trafugato in casa dell'anziano e mal messo Cornelius Gurlitt un tesoro d'arte realizzato dai grandi artisti la cui opera negli anni della dittatura era marchiata come "degenerata". Sono i principali esponenti del modernismo: Pablo Picasso, Henri Matisse, Marc Chagall, Emil Nolde, Franz Marc, Paul Klee, Oskar Kokoschka, Ludwig Kirchner e Max Liebermann. Le opere in questione furono regolarmente acquistate dal padre di Cornelius, Hildebrand Gurlitt (18951956), commerciante d'arte e critico, voluto da Hitler in persona come acquirente all'estero di opere d'arte moderna e d'avanguardia "diffamanti", perché fossero 
consegnate e distrutte, e, dall'inizio della Seconda guerra mondiale, come scout e fidato acquirente di opere, sempre per conto del Führer stesso, da conservare a Linz nel futuro - e mai realizzato - Hitlermuseum, su progetto di Albert Spear. Hildebrand Gurlitt rappresenta la chiave di volta nell'intera questione, perché da un lato è il segugio sguinzagliato, ma da un altro lato è colui, che si oppose alla distruzione, alla damnatio memoriae dell'avanguardia, acquistando con soldi propri a famiglie ebree, nascondendo e salvaguardando così - se non le vittime più importanti - un tassello d'arte di carattere epocale, considerando i soggetti citati. La natura del "bottino" riesumato in Baviera è di questa congerie.

Oblio dunque per il singolo artista come per il singolo condannato al gas, al fine di corrodere la storia collettiva; e se si volevano distruggere milioni di persone, per il nazifascista, significava riuscire anche a cancellare con la singola storia la denuncia che l'avrebbe dilagata nel tempo: la lungimirante sconfessione sancita dall'arte d'avanguardia, estinguendo le radici civili e religiose europee per scrivere una storia posticcia. Una storia, infatti, che in assenza di tracce precedenti sarebbe partita da zero, imposta in un alfabeto nuovo, che con la cultura occidentale e la tradizione avrebbe condotto percorsi selettivi come si fece de facto in politica razziale. Il futuro nazista, se questo piano fosse andato a segno, sarebbe oggi un ibrido increscioso, un presente degno di un passato vivisezionato, blindato tra roghi e distruzioni tormentate da omelie savonaroliane, da libri bruciati in piazza tra rituali ossessi, mentre i crematori smaltivano gasati.

Un ritualismo metastorico in cui trova totale conferma l'assunto secondo il quale il nazifascismo abbia ereditato dal passato, ripresentando percorsi noti, con il risultato ben più agghiacciante, dato dalla continuità culturale implicita nel provvedimento stesso della damnatio memoriae. Un'adamantina continuità tra ere, che ha voluto dunque dipanarsi con strumenti culturali noti e impressi nella genealogia del disprezzo, attraverso un altisonante parallelo culturale, di auto consacrazione ai destini della storia, attivato in poche parole, dal processo di damnatio memoriae nell'arte stessa; per sciogliere nel nulla il contrappunto dialettico di un'arte che sconfessasse intellettualmente, attraverso proprie vie d'indagine, i deliri di onnipotenza e il modo populista e kitsch del fascismo di congelare le vie della storia nel vacuum ariano. Un'arte che parlasse di evoluzioni politiche, di recrudescenze ideologiche e sociali, nonché di ripudio della guerra, non poteva appartenere ai percorsi visivi culturali della storia dell'arte tedesca, sulla via appunto del pangermanesimo. 


\section{Damnatio memoriae: una lunga storia}

Un'assenza, dunque, al cui posto, grazie a personaggi come Hildebrand Gurlitt, in Germania sarebbero dovute affluire nuove presenze artistiche di regime, in apoteosi sulla damnatio memoriae precorsa. Parlare di damnatio memoriae in fondo è entrare nel vivo della storia dell'arte e della politica. E quale paese europeo più dell'Italia potrebbe mai esimersi dal prenderne atto? Damnatio memoriae è "condanna della memoria", per la totale cancellazione delle tracce memoriali a causa di un profondo disprezzo: un buco documentario creato ad hoc per distruggere il ricordo, per impedire il percorso a ritroso sui pioli originali di una storia, in totale assenza di cronaca.

La damnatio memoriae è foriera di absentia, è un gesto politico in circolazione fin dai tempi dell'antica Roma per osteggiare i nemici a tal punto che all'opposto dell' apoteosi la vittima designata, condannata alla damnatio era come se non fosse mai esistita nella storia di Roma. Centro culturale di un popolo che aveva affidato all'immagine i cardini fondamentali del suo stesso onore, una tendenza che appunto ha permeato gli strati storici dei secoli toccando nazismo e fascismo e l'oggi più che mai, nel Gotha morboso del nostro bisogno sociologico di apparire grazie a tecnologie invadenti: tv, internet (con i vari social network) e il cinema. Così orgoglio e reputazione, con la damnatio memoriae, da Roma in poi svaniscono: un'atroce condanna alla dimenticanza, se radicata in un ambiente nel quale essere e appartenenza sono elementi di vanto. In età repubblicana (509-27 a.C.), quando per quasi mezzo millennio Roma ebbe un senato, alla lista delle pene che potevano essere inflitte - anche a un sovrano - si aggiunse l'abolitio nominis: il prenome del condannato doveva essere totalmente cancellato dalle iscrizioni pubbliche e anche dentro la sua famiglia non poteva essere più tramandato. E non finiva qui, spesso si giungeva a estremi più radicali, come la recissio actorum: la totale distruzione di tutte le opere realizzate dal condannato stesso. Quando il reo era ancora vivo, quel che seguiva da questi provvedimenti straordinari era una vera e propria morte civile.

Questa è la fine di Massimino Trace e del figlio Gaio Giulio Vero Massimo, che fu Cesare. Si trovavano alla frontiera danubiana quando nel 238 d.C. il senato dichiarò Massimino nemico dello stato. Massimino e Massimo si mossero allora verso l'Italia, ma Aquileia gli chiuse le porte, ponendoli sotto assedio. Le truppe, tra fame e malattie, gli divennero ostili. Così presi dal timore, durante un momento di pausa, strapparono le immagini di Vero Massimo dalle insegne militari, per segnalare la sua deposizione, per poi assassinarlo nel suo accampamento, assieme al padre Massimino, mentre erano coricati sotto la tenda. Poi infilate le teste in 
cima alle picche, ne fecero mostra agli Aquileiensi. A Roma allora furono subito abbattute le sue statue e i suoi busti, mentre il suo prefetto del pretorio era assassinato con altri suoi amici. Poi le teste dei due ex-sovrani, padre e figlio, furono inviate nell'Urbe, mentre i loro corpi mutilati furono dati in pasto ai cani. Il senato ordinò la damnatio memoriae per Massimino [Verner 2004, 202-3].

Da Roma in poi la damnatio memoriae lungi dal semplificarsi, ci coinvolge in capitoli altrettanto inquietanti, profilandosi incisiva oltre la latenza di una seduzione totalizzante e ammantata d'estetica, descrivendo ampi archi di storia umanistica. Nel Medioevo colpì perfino la Roma papalina, come nel caso di Papa Formoso, il cui "sinodo del cadavere", terribile e inquietante, è degno di un racconto di Edgar Allan Poe. Formoso morì in contumacia (896) con a suo carico ben sette gravi accuse prettamente politiche. Gli successe, dopo il brevissimo pontificato (15 giorni) di Bonifacio VI, Papa Stefano VII, che fece riesumare il cadavere di Formoso, imbellettare con i paramenti pontifici per poi condurlo al processo: pur morto doveva rispondere seduto sul trono a tutte le accuse accumulate quando "smodato" e "ambizioso" tramò contro i santi equilibri del soglio pontificio. La condanna al cadavere fu esemplare: tutti i suoi atti furono annullati; i suoi ordini in vita invalidati, le vesti papali - al pubblico ludibrio del cadavere - gli furono strappate di dosso, le tre dita pontificie della mano destra usate per la benedizione

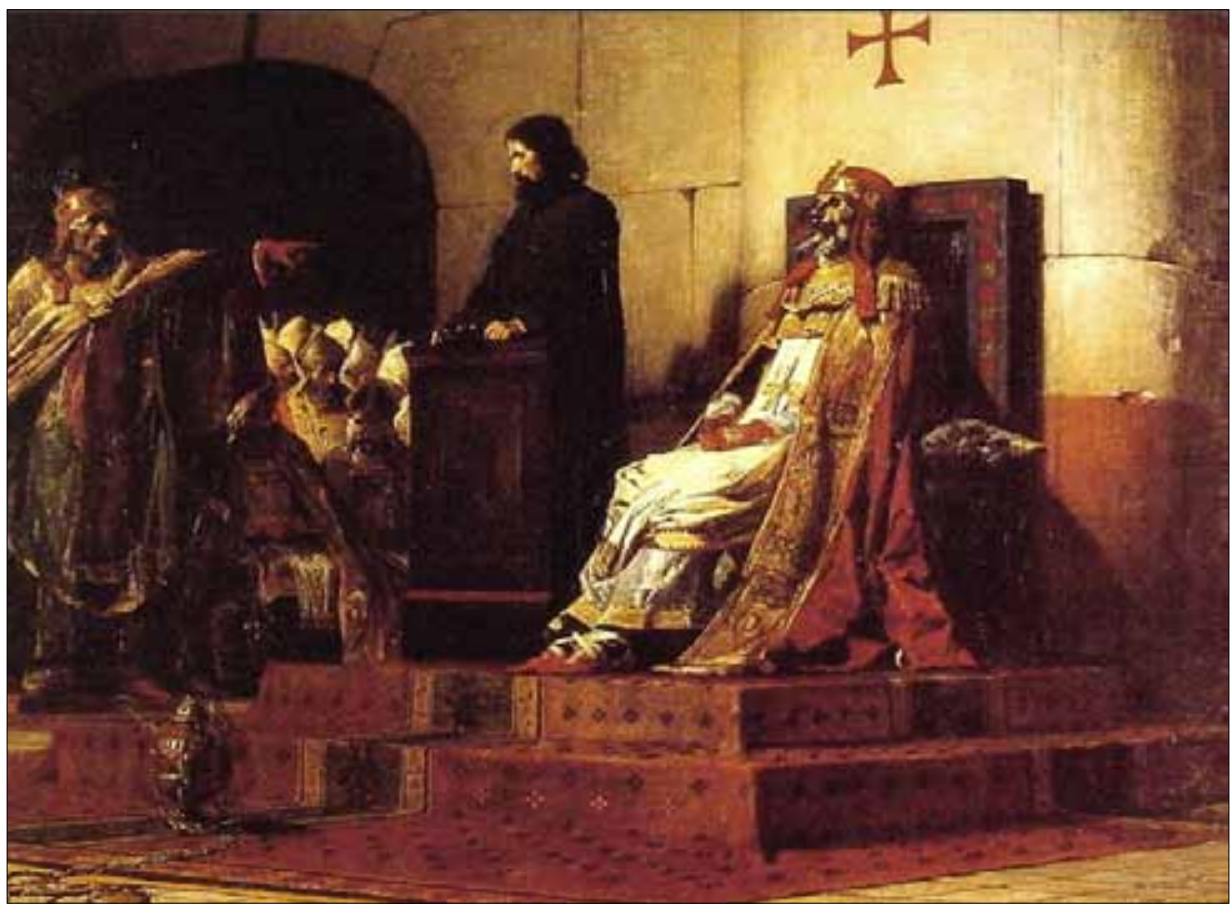

Jean-Paul Laurens, Le Pape Formose et Etienne VII (1870) 
gli furono tagliate e tra urla beduine il cadavere fu trascinato via dalla sala del processo e gettato nel Tevere. Tutte le opere o effigi furono ritirate e nessuno avrebbe mai più potuto nominare Papa Formoso.

Un grande artista francese del periodo chiamato "art pompier", il parigino JeanPaul Laurens (1838-1921), non a caso negli anni in cui l'arte scivolava tra simbolismo e immaginifico, realizzò il suo più celebre dipinto Le Pape Formose et Etienne VII (1870).

Adolf Hitler captò il potenziale ipnotico e la sospensione presente in questo tipo di produzione inquietante, eleggendo propria icona un artista contemporaneo del Laurens, autore di una produzione ben più morbosa e emblematica, lo svedese Arnold Böklin (1827 - 1901).

Un altro capitolo di rilievo nei percorsi della damnatio memoriae soprattutto per l'arte stessa, e il suo valore politico, è la vicenda del Doge Marin Faliero (12851355), che fu il cinquantesimo doge veneziano deposto e giustiziato per alto tradimento. La congiura contro Venezia ordita da Faliero fu provata dal procuratore padovano Nicolò Lion che arrestò i complici del Faliero, costringendoli a tradire l'orditore. Arrestato e torturato, Marin Faliero confessò davanti al Tribunale dei dieci che all'unanimità lo condannò alla pubblica decapitazione, in piazzetta San Marco il 17 aprile del 1355. Tutto il popolo veneziano a occhi aperti vide la decapitazione e la spada insanguinata del boia che, si dice, urlò a squarciagola: «Vardé tuti! L'è stá fata giustizia del traditor!». Da questo episodio, come nel caso di Papa Formoso, le arti visive restarono profondamente colpite e due grandi artisti ci hanno lasciato due opere memorabili del momento della decapitazione: Eugène Delacroix con L'esecuzione di Marin Falier (1827) e Francesco Hayez con Gli ultimi momenti del Doge Marin Falier (1867).

\section{Ritorno al '900 nazista}

E se come visto col passare dei secoli tali provvedimenti furono inflitti a presunti o veri criminali, il boomerang della storia non ha risparmiato quegli stessi criminali del nazismo e del fascismo, che oltre a sterminare si arrogarono il diritto di dannare, ripagandoli con la stessa moneta. Il tentativo proposto dal nazifascismo di assenza forzata è risultato difatti (come questa collettiva si propone di rimarcare) rivitalizzante se pur in absentia delle presenze, con produzioni d'arte empatica che valica i limiti dati dal tempo, oltre i testimoni stessi. A quei criminali del nazifascismo è andata molto peggio perché a costoro, più che la damnatio memoriae, è toccata invece la dannazione della memoria per un'intera epoca e per l'ideologia stessa. Quel che per i nazisti rimasti deve essere stata la più atroce 


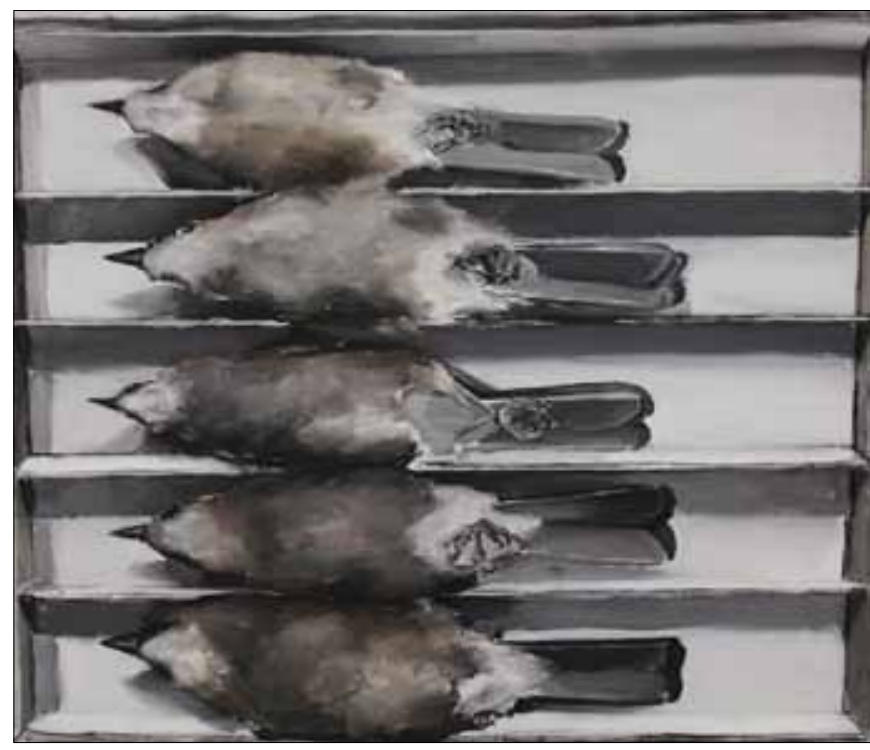

Dalla mostra In Absentia: Santiago Ydañez, Senza titolo (2006)

delle pene da subire.

Magda Goebbels si ammazzò - dopo aver ucciso l'intera prole - perché non poteva immaginare un mondo senza il nazionalsocialismo.

Hermann Göring, capo supremo della Luftwaffe tedesca processato a Norimberga, e condannato a morte per i suoi crimini, si tolse la vita nel 1946 prima che la sentenza fosse

eseguita. Il motivo del suicidio è l'ultimo gesto di un nazista integrale depresso e sconfitto da ciò che a processo gli sussurrò, all'ennesimo ghigno sprezzante, durante uno degli interrogatori, uno dei giovani avvocati attendenti in assise. Il frangente fu emblematico (la seduta venne improvvisamente sospesa), come la natura del messaggio inflitto: gli si fece chiaramente intendere che per i giudici tutti e ventiquattro gli imputati erano considerati già appesi alla forca, perché di prove a loro carico ce n'erano fin troppe; ciò a cui quegli interrogatori servivano, tenerli ancora in vita serviva a capire che cosa fosse realmente stato il nazionalsocialismo, si voleva carpirne il nocciolo più profondo, in modo da garantire al mondo la fine totale del nazismo e di un'epoca aberrante, per costituire una società post-nazista totalmente antitetica e rieducata, in una totale dannazione dell'ideologia, perché non ne restasse un solo mattone in piedi.

A prescindere dai risultati, archivi e musei per fortuna pullulano di tracce documentarie e prove tangibili sul nazifascismo. Un deterrente almeno è posto. Tuttavia è rimarchevole la cancellazione dei simboli legati al nazismo in Germania, per esempio, dal tessuto civile come anche della rappresentazione (recissio actorum) proprio per contravvenire a ogni possibile presenza di tracce estetiche continuamente sotto gli occhi, tendendo sempre a connotare con la locuzione "indelebili cicatrici" l'inamovibile. Scivolando nel particolare, poi, quei volti del nazismo intrisi di vanagloria e auto celebrazione, ritratti da artisti compiacenti, sono negati alla vista del pubblico. Anche a essi è negata luce e si sono spenti nella memoria 
di chi li ha visti. Gli scantinati dei musei tedeschi sono stipati di opere che per legge non possono essere esposte in patria o all'estero. Ci sono burocrati e critici sguinzagliati per rinvenirne le tracce e una legge categorica che ha totalmente galvanizzato la sempiterna e sibillina fiammella dell'ideologia, troppo vicina alla natura umana, sempre in agguato, perché basta poco a far divampare l'incendio dell'orgoglio nazionale, che fu il primo ingrediente dell'ideologia fascista.

Così i ritratti eroici di Hitler, Goebbels e dei vari gerarchi - dipinti sulla scia

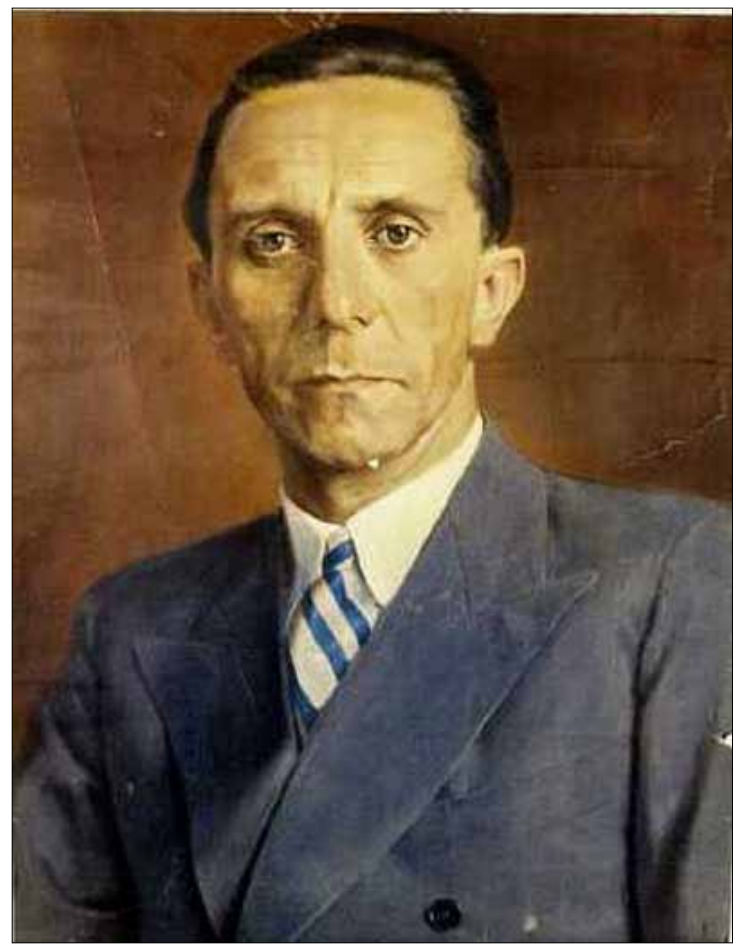

Wilhelm Otto Pitthan, Ritratto di Goebbels (1942) degli imperatori e cesari romani - restano tra le macerie stesse della storia, ma comunque intatti e irrintracciabili in una iconoclastia al contagocce, che vieta categoricamente il venir meno della damnatio memoriae. Tant'è che possono circolare solo alcune immagini di Hitler \& Co.: in Germania sono sempre le stesse, usate più che altro dalla stampa accreditata, che continua a prodursi (deve) in articoli e reportage tesi a scardinare dalla storia del nazionalsocialismo ogni alterezza gettandola nello squallore vissuto dai tedeschi alla fine del sogno. Pena il divieto di pubblicazione...

A questo fondo di tanti e troppi ritratti, eseguiti con dovizie mnemonica e ogni cura nei particolari per il messaggio ai posteri, spetta la pena del buio eterno, sepolti vivi, mentre sopra di loro la società tedesca continua nei suoi sforzi: ritrovare le opere "degenerate" per catalogarle, che valgono miliardi e pesano di cultura; ricostruire e ricordare ciò che la menzogna della razza è costata: infamia, disprezzo, distruzione. Del resto anche i cadaveri dei gerarchi nazisti cadono in damnatio memoriae, non si possono seppellire in cimiteri civili né tantomeno ricevere lapidi o scritte commemorative. Valga per tutti il recente caso di Erich Priebke (1913-2013) la cui sepoltura è stata oggetto di polemiche e riprese tra Italia e Germania. 


\section{II caso italiano}

La stessa cosa, con risultati spesso mediocri, e tipicamente goffi, è stata appunto tentata anche in Italia, più che altro in termini giuridici, come la conventio ad excludendum: locuzione latina coniata in tempi moderni dal giurista e politico Leopoldo Elia quando denunciò negli anni Settanta il così detto "pentapartito", che si intestardì nel non considerare il Pci come una forza politica e democratica al governo, rifiutando quindi ogni possibile politica interlocutoria, perché il comunismo italiano sarebbe stato troppo coinvolto in amicizie e pendenze con l'Unione sovietica.

Anche per il partito neo fascista dell'Msi fu applicata la conventio ad excluden$d u m$. Difatti fu escluso anch'esso per decenni non solo da un accesso al governo, ma anche da qualsivoglia collaborazione o dialogo con altre forze politiche, a causa del legame troppo stretto tra questo partito e il disciolto partito fascista. In questo caso il tacito accordo di esclusione era formalizzato dall'espressione "arco costituzionale", che includeva tutte le forze politiche (Pci compreso) che avevano partecipato alla Resistenza antifascista e alla stesura della Costituzione, e teneva fuori i neofascisti.

Altro caso invece, sempre in ambito politico, è quello della conventio ad tacen$d u m$, che appunto è un accordo esplicito o una tacita intesa tra alcune parti sociali, economiche o politiche, che abbia come fine il tacere su una particolare circostanza, dato o realtà. Assume spesso funzioni di autodifesa: una minoranza, ad esempio, evita di porre l'accento o tace gli aspetti di diversità rispetto alla maggioranza, allo scopo di non suscitare sospetti o risentimenti. Può essere ad esempio il caso di ebrei o cristiani in paesi musulmani. Nella nostra storia, si trovano anche esempi in campo politico: nella Spagna di Francisco Franco, ad esempio, era un titolo di merito per una persona o un'azienda (fino al 1943) essere definiti italiani. Dopo, appunto, era più opportuno evitare di citare questa circostanza, che sarebbe stata svantaggiosa.

«Nell'Italia successiva alla seconda guerra mondiale, invece, per alcuni decenni fu particolarmente colpita da questa esclusione di menzione la città di Forlì, percepita - essendo stata "città del Duce" - come uno dei simboli del passato regime fascista: in sostanza, tutte le volte che non fosse proprio inevitabile citarla, Forlì non doveva essere nemmeno nominata» [Landi s.d.]. Giorgio Bocca, negli anni Sessanta, riferendosi al caso di Forlì, usa anche l'espressione "complesso del Duce" [Balzani 2011]. La conventio ad tacendum, come si vede, può presentare, ma solo per alcuni aspetti, qualche somiglianza con la damnatio memoriae, ma se 
ne distingue poiché può essere spesso volontariamente accettata o perfino voluta da coloro stessi a cui si applica.

Quel che è mancata in Italia è stata la determinazione nel cancellare la presenza incombente di busti, teste, pubblicazioni in memoria stessa di Mussolini, in tutti i sondaggi sempre ai primi posti nell'immaginario nazionale tra gli italiani più apprezzati, con Leonardo e Michelangelo. I mercatini delle pulci romani, e non, gongolano di effigi del fascismo in vendita, i calendari con Mussolini in posa, mentre retore parla al popolo, sono sotto gli occhi di tutti, appesi in bella vista nelle edicole cittadine in molte regioni. E se tutto ciò che può essere appeso è arte, sosteneva il dadaismo, è anche vero che l'estetica del fascismo in Italia non è mai stata alienata, anzi ipocritamente fatta circolare, mai staccata per essere protratta tra trasgressione e volontà d'affermazione.

In effetti, il fascismo è fin troppo radicato all'immagine di Roma come la damnatio memoriae, baluardo l'uno, e purga aristocratica l'altra, della cultura italiana, ma in un processo d'identificazione sbagliato, perché riproposizione kitsch di vecchi fasti. Non ha portato a esiti encomiabili il sentirsi Ottaviano Augusto, come faceva Mussolini, quando parlava della grande Roma repubblicana (in verità del principato); come a altrettante disfatte morali approda il popolo quando sente il dolce gusto d'immedesimazione in certe rimembranze, di continuità tra Roma antica e oggi, tra i grandi cesari e il Duce.

E ciò avviene in Italia perché, a differenza della Germania, qui non potendo andar fieri di un certo presente politico e della rarefazione culturale, si ricerca legittimazione nel passato senza vergognarsi con tono interrogativo alla voce "fascismo". Perché, ancora a differenza della consorella Germania, in Italia si è solo pacificato, per non vivere il disagio delle vampate d'imbarazzo [Trapani 2010]. Attraverso artisti contemporanei di grande calibro A.R.S. - Art Resistance Shoah, lungi dal voler rimarginare la ferita della memoria pacificando, continuerà a offrire la frattura, caustico e empatico, in nome della presunzione di colpevolezza, perché si riempia quel vuoto di assenze ricordate ma raramente onorate in un paese che ha raggiunto apici epocali nel dramma dell'arte come in conventio ad tacendum. 


\section{Bibliografia}

Balzani R. 2011, La Forli di Giorgio Bocca, "InformaForli”, 27 dicembre.

Landi M. s.d., Propaganda e antipropaganda. Il caso Forli, “l’Idea”, http://digilander. libero.it/idea.ap/landi.htm.

Trapani S. 2010, Auschwitz e la sua memoria, "Ricerche storiche", 110.

Verner E. 2004, Mutilation and Transformation: Damnatio Memoriae and Roman Imperial Portraiture, Leiden: Brill.

\section{Risorse}

Comune di Correggio http://www.comune.correggio.re.it/servizi/notizie/notizie_homepage.aspx

Correggio Art Home http://www.correggioarthome.it/

Gemäldegalerie - Staatliche Museen zu Berlin http://www.smb.museum/home.html

Istoreco - Istituto per la storia della Resistenza e della società contemporanea in provincia di Reggio Emilia http://www.istoreco.re.it/ 